\title{
Horizontally Transferred Genetic Elements in the Tsetse Fly Genome: An Alignment-Free Clustering Approach Using Batch Learning Self-Organising Map (BLSOM)
}

\author{
Ryo Nakao, ${ }^{1,2}$ Takashi Abe, ${ }^{3}$ Shunsuke Funayama, ${ }^{3}$ and Chihiro Sugimoto ${ }^{4,5,6}$ \\ ${ }^{1}$ Unit of Risk Analysis and Management, Hokkaido University Research Center for Zoonosis Control, Kita 20, Nishi 10, \\ Kita-ku, Sapporo, Hokkaido 001-0020, Japan \\ ${ }^{2}$ Laboratory of Parasitology, Department of Disease Control, Graduate School of Veterinary Medicine, Hokkaido University, \\ Kita 18, Nishi 9, Kita-Ku, Sapporo, Hokkaido 060-0818, Japan \\ ${ }^{3}$ Graduate School of Science \& Technology, Niigata University, No. 8050, Igarashi 2-no-cho, Nishi-ku, Niigata 950-2181, Japan \\ ${ }^{4}$ Division of Collaboration and Education, Hokkaido University Research Center for Zoonosis Control, Kita 20, Nishi 10, \\ Kita-ku, Sapporo, Hokkaido 001-0020, Japan \\ ${ }^{5}$ Global Station for Zoonosis Control, Global Institution for Collaborative Research and Education (GI-CoRE), \\ Hokkaido University, Kita 20, Nishi 10, Kita-ku, Sapporo, Hokkaido 001-0020, Japan \\ ${ }^{6}$ Department of Disease Control, School of Veterinary Medicine, University of Zambia, P.O. Box 32379, Lusaka, Zambia
}

Correspondence should be addressed to Chihiro Sugimoto; sugimoto@czc.hokudai.ac.jp

Received 30 June 2016; Revised 26 September 2016; Accepted 8 November 2016

Academic Editor: Farit M. Afendi

Copyright ( 2016 Ryo Nakao et al. This is an open access article distributed under the Creative Commons Attribution License, which permits unrestricted use, distribution, and reproduction in any medium, provided the original work is properly cited.

Tsetse flies (Glossina spp.) are the primary vectors of trypanosomes, which can cause human and animal African trypanosomiasis in Sub-Saharan African countries. The objective of this study was to explore the genome of Glossina morsitans morsitans for evidence of horizontal gene transfer (HGT) from microorganisms. We employed an alignment-free clustering method, that is, batch learning self-organising map (BLSOM), in which sequence fragments are clustered based on the similarity of oligonucleotide frequencies independently of sequence homology. After an initial scan of HGT events using BLSOM, we identified 3.8\% of the tsetse fly genome as HGT candidates. The predicted donors of these HGT candidates included known symbionts, such as Wolbachia, as well as bacteria that have not previously been associated with the tsetse fly. We detected HGT candidates from diverse bacteria such as Bacillus and Flavobacteria, suggesting a past association between these taxa. Functional annotation revealed that the HGT candidates encoded loci in various functional pathways, such as metabolic and antibiotic biosynthesis pathways. These findings provide a basis for understanding the coevolutionary history of the tsetse fly and its microbes and establish the effectiveness of BLSOM for the detection of HGT events.

\section{Introduction}

Tsetse flies (Glossina spp.) are the primary vectors of trypanosome parasites; they cause human African trypanosomiasis (or sleeping sickness) and animal African trypanosomiasis (or nagana) in Sub-Saharan African countries. The flies harbour three maternally transmitted endosymbionts, Wigglesworthia glossinidia, Sodalis glossinidius, and Wolbachia pipientis, which influence host physiology. For example, Wigglesworthia provides essential nutrients, such as vitamins, to the host [1,2] and influences host immune maturation [3]. Although the precise role of Sodalis in the tsetse fly is not clear, it appears to influence various host properties, such as longevity and susceptibility to trypanosome infections [46]. In many arthropod species $[7,8]$, Wolbachia induces strong cytoplasmic incompatibility, which was also observed in the tsetse fly [9]. In addition to these common bacteria, a recent microbial population analysis using a deep-sequencing approach revealed other facultative microorganisms from diverse bacterial families in the guts of tsetse flies, though 
their relative abundances were very low compared to that of the symbiont Wigglesworthia [10].

In addition to the parasitism of bacterial organisms themselves, partial genome sequences of Wolbachia are incorporated into the tsetse fly genome. Initially, Doudoumis et al. reported the incorporation of short fragments of three Wolbachia genes (16S rDNA, fbpA, and wsp) in the genomes of laboratory and natural Glossina morsitans morsitans $(\mathrm{Gmm})$ populations [11]. Subsequently, a whole-genome sequencing project revealed large insertions of the Wolbachia genome in the Gmm genome via horizontal gene transfer (HGT) events $[12,13]$. These insertions were identified by extracting Wolbachia-specific sequences from whole-genome Sanger sequencing reads and pyrosequencing data based on nucleotide homology with the complete genome sequences of three Wolbachia strains (wMel, wRi and wBm) [13]. Fluorescent in situ hybridisation analyses further confirmed the presence of these insertions in Gmm on the two sex chromosomes ( $\mathrm{X}$ and $\mathrm{Y}$ ) and the supernumerary B-chromosome $[12,13]$.

HGT elements can be detected by two main methods: phylogeny-based and composition-based methods [14]. The first method relies on sequence alignments; HGT is identified when the position of a query sequence in a tree does not match that of a reference phylogeny. Although this approach is robust, the frequency of HGT events may be underestimated, especially when there is a lack of information on donor sequences [15]. The second method relies on nucleotide compositional features, such as $\mathrm{G}+\mathrm{C}$ content, nucleotide frequencies, or codon usage [16-18], and theoretically does not require sequence homology. Batch learning self-organising map (BLSOM) is an alignment-free clustering method that generates a map independently of the order in which data are input via a learning process $[19,20]$. This method enables the clustering of genomic sequence fragments based on the similarity of oligonucleotide frequencies, without any other taxonomical information; it has been successfully applied in genomic and metagenomic studies [20-22].

The objective of this study was to characterise HGT from microorganisms in the genome of $\mathrm{Gmm}$ using the alignmentfree clustering method BLSOM. Using BLSOM, we detected a number of HGT candidates from diverse origins. In a comparison of the results for HGT from Wolbachia between methods, there was a high level of agreement between BLSOM and BLASTn, a homology-based approach. Based on functional annotation, these potential HGT elements encoded loci in various functional pathways.

\section{Materials and Methods}

2.1. Genome Sequences. The tsetse fly $(\mathrm{Gmm})$ genome (Accession number CCAG010000000) and all prokaryotic sequences identified to the species level $(n=5,600)$ were obtained from GenBank (http://www.ncbi.nlm.nih.gov/ Genbank/). When the number of undetermined nucleotides (Ns) exceeded $10 \%$ of the window size $(5 \mathrm{~kb})$, the sequence was omitted from the analysis. When the number of Ns was less than $10 \%$, the oligonucleotide frequencies were normalised to the length without Ns and included in the analysis.

2.2. Batch Learning Self-Organising Map. G+C\% is a fundamental value for the phylogenetic classification of microbial genomes, including viral genomes, but it cannot differentiate a wide variety of genomes. Oligonucleotide composition can distinguish species, even those with the same $\mathrm{G}+\mathrm{C} \%$, because it varies substantially among genomes; accordingly, it is referred to as a "genome signature" [23]. Multivariate analyses, such as factor correspondence analysis and principal component analysis (PCA), are useful to investigate variation in gene sequences [24]. However, the clustering power of conventional multivariate analyses is inadequate when massive quantities of sequence data from a wide variety of genomes are analysed collectively. Kohonen's selforganising map (SOM) is a powerful tool for clustering and visualising high-dimensional data vectors on a twodimensional plane $[25,26]$. To handle codon and oligonucleotide composition as high-dimensional data vectors, we modified the conventional SOM to develop the BLSOM [19, 20], which is suitable for genome sequence analyses and highperformance parallel computing. The initial weight vectors were defined by PCA, instead of random values, based on the finding that PCA can classify gene sequences into groups of known biological categories. Weight vectors $\left(\mathbf{w}_{i j}\right)$ were arranged in the two-dimensional lattice denoted by $i(=0$, $1, \ldots, I-1)$ and $j(=0,1, \ldots, J-1)$. Weight vectors $\left(\mathbf{w}_{i j}\right)$ were set and updated as described previously [19, 27]. A BLSOM program suitable for PC cluster systems is available on our website (http://bioinfo.ie.niigata-u.ac.jp/?BLSOM).

\subsection{Detection of HGT Candidates in the Tsetse Fly Genome} and Prediction of Their Origins Using BLSOM. To identify HGT candidates in the tsetse fly genome derived from prokaryotes, two types of large-scale BLSOM were used, that is, Tsetse+Prokaryotes- and Genus-BLSOM, using all genome sequences deposited in DDBJ/ENA/GenBank. A Tsetse+Prokaryotes-BLSOM was constructed with a degenerate tetranucleotide composition for all $5 \mathrm{~kb}$ sequences derived from tsetse fly genome sequences of longer than $5 \mathrm{~kb}$ plus 5,600 identified prokaryotes for which at least $10 \mathrm{~kb}$ of sequence was available from DDBJ/ENA/GenBank. The degenerate tetranucleotide composition was the composition of degenerate sets in which a pair of complementary tetranucleotides was added (e.g., ATGC and GCAT). To obtain more detailed phylotype information for the prokaryotic sequences, Genus-BLSOM was constructed for each phylum derived from 5,600 identified prokaryotes.

For tsetse fly contigs of longer than $5 \mathrm{~kb}(9,710$ contigs), a $5 \mathrm{~kb}$ window with a $1 \mathrm{~kb}$ step was used to obtain 303,250 segments (Figure 1, Step 1), which were mapped to Tsetse+Prokaryotes-BLSOM by identifying the lattice point with the minimum Euclidian distances in the multidimensional space (Figure 1, Step 2). For every lattice point at which tsetse fly genomic segments were mapped to prokaryotic territories, the most abundant phylum was identified, and 


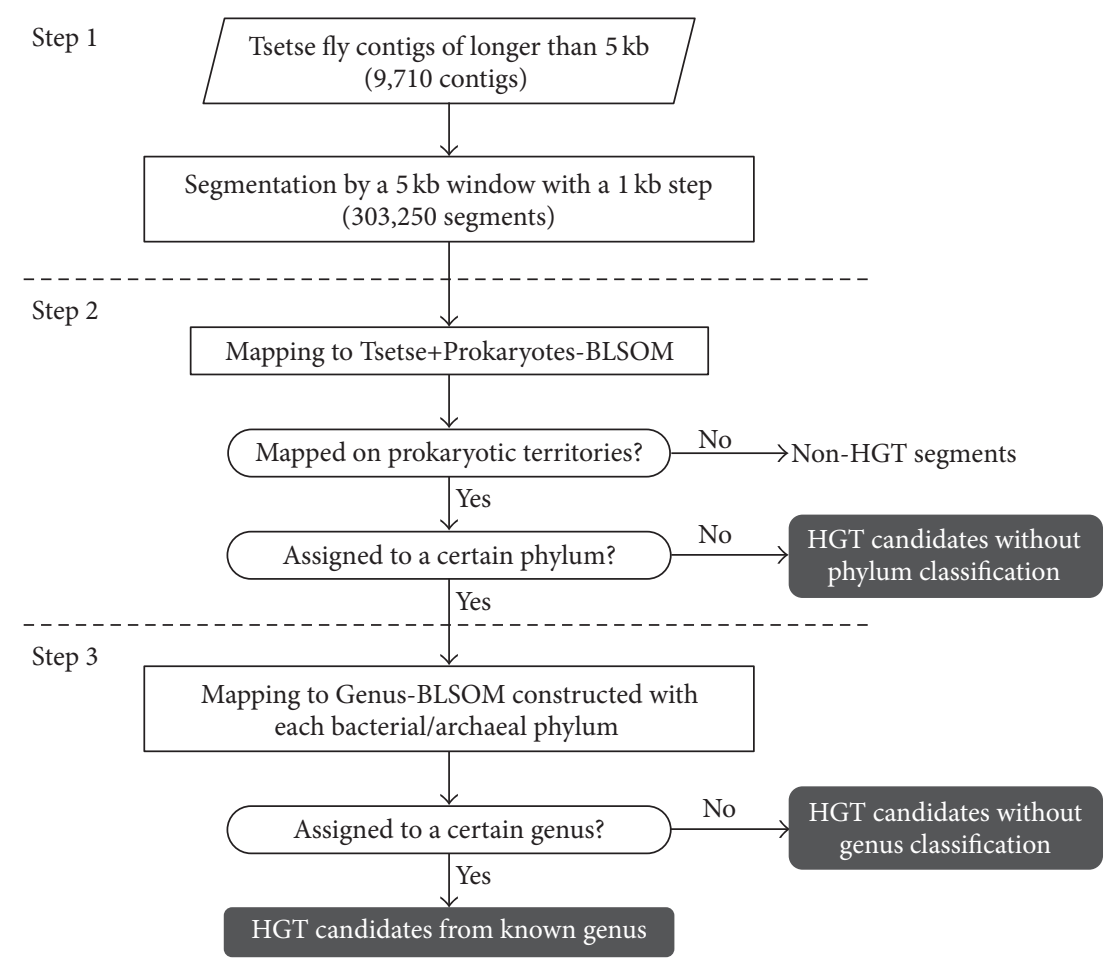

FIgURE 1: Workflow for data processing and BLSOM analysis.

the mapped tsetse fly genomic segments were tentatively assumed to belong to the phylum. Finally, when the most abundant phylum in more than $40 \%$ of the segments derived from a single tsetse fly contig was the same, the tsetse fly contig was assigned to this phylum by BLSOM. To identify the phylogenetic origin of the tsetse fly genomic segments that were mapped to the prokaryotic territories on Tsetse+Prokaryotes-BLSOM, they were successively mapped on Genus-BLSOM (Figure 1, Step 3). Similar stepwise mappings of tsetse fly genomic segments on BLSOMs constructed with sequences from more detailed phylogenetic categories (e.g., genera) were conducted.

2.4. Detection of HGT Candidates by BLASTn. To detect HGT candidates derived from Wolbachia, a local BLASTn search was conducted with the contig sequences of the tsetse fly that exceeded $5 \mathrm{~kb}$ against the NCBI nonredundant nucleotide database. When more than $1 \mathrm{~kb}$ of the sequence showed similarity with Wolbachia with top-hits and an Evalue threshold of $1 \times 10^{-5}$, the contigs were considered HGT candidates derived from Wolbachia.

2.5. Functional Classification of HGT Candidates. The sequence fragments with prokaryote origins were functionally annotated using KEGG (Kyoto Encyclopedia of Genes and Genomes) mapping [28] with the KAAS web server (http://www.genome.jp/tools/kaas/) [29]. KEGG Orthology $(\mathrm{KO})$ assignments were obtained using the single-directional best-hit method. The organisms included in the analysis were as follows (based on IDs): hsa, dme, ath, sce, pfa, eco, sty, hin, pae, nme, hpy, rpr, mlo, bsu, sau, lla, spn, cac, mge, mtu, ctr, bbu, syn, aae, mja, afu, pho, and ape. The organisms in the database are listed on the KAAS web server (http://www.genome.jp/kaas-bin/kaas_org).

\section{Results and Discussion}

3.1. Detection of HGT Candidates Using BLSOM. Of 303,250 sequence segments obtained from the tsetse genome, we found that 11,524 sequences $(3.8 \%)$ clustered with reads from prokaryotes and thus were HGT candidates according to Tsetse+Prokaryotes-BLSOM (Figure 2). These sequences were distributed across 2,960 different contigs, corresponding to $30.48 \%$ of all contigs. We assigned the most sequences to the phylum Firmicutes $(n=758)$, followed by the phyla Bacteroidetes $(n=370)$, Alphaproteobacteria $(n=90)$, and Gammaproteobacteria $(n=23)$ (Table 1$)$. We did not assign 1,671 contigs to phyla owing in part to the presence of HGTs from multiple phyla within the same contig. It is also possible that these candidates were introduced by ancient HGT events, and their oligonucleotide compositions drifted over time [30], limiting the use of composition-based methods for classification.

We performed further characterisation of donor sequences to the genus level using Genus-BLSOM (Figure 3). The results of four dominant phyla are summarised in Table 2 . We assigned the most sequences to the genus Bacillus ( $n=$ 239). The second most highly represented origin was the class Flavobacteria $(n=187)$, which we were unable to classify to the genus level owing to coclustering with genome sequences that lacked genus information, followed by the genera 


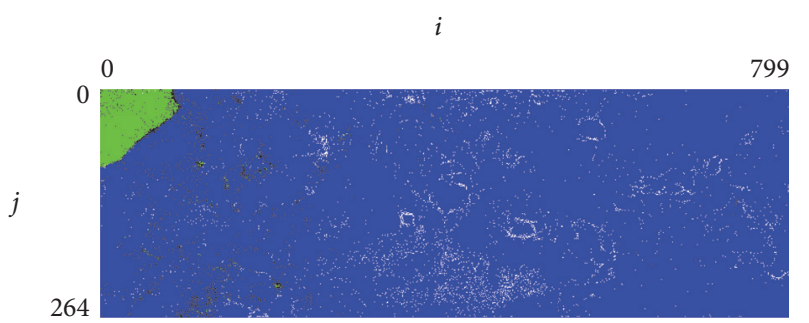

(a)

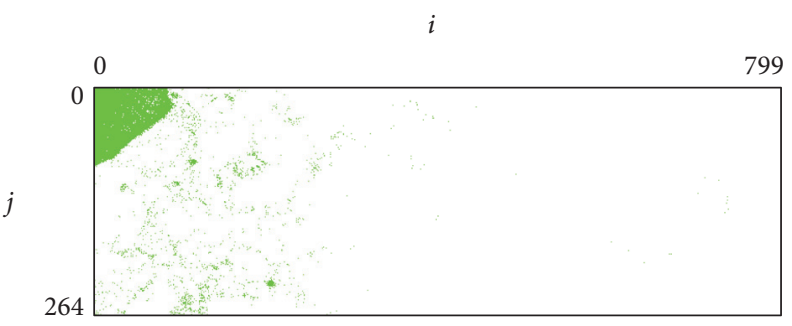

(b)

FIGURE 2: Tsetse+Prokaryotes-BLSOM. (a) BLSOM using the degenerate tetranucleotide set for the tsetse fly plus 5,600 identified prokaryotes. Lattice points that include the sequences from the tsetse fly are indicated in green, those that contain no genomic sequences are indicated in white, and those containing sequences from a prokaryote are indicated in blue. Lattice points that include both tsetse fly- and prokaryotesequences are shown in black. (b) Distribution of tsetse fly genome sequences. Only green lattice points are shown.

TABLE 1: Origins of HGT candidates at the phylum level.

\begin{tabular}{lc}
\hline Phylum & Number of contigs \\
\hline Actinobacteria & 10 \\
Alphaproteobacteria & 90 \\
Aquificae & 2 \\
Bacteroidetes & 370 \\
Betaproteobacteria & 4 \\
Crenarchaeota & 1 \\
Cyanobacteria & 1 \\
Epsilonproteobacteria & 16 \\
Euryarchaeota & 12 \\
Firmicutes & 758 \\
Fusobacteria & 1 \\
Gammaproteobacteria & 23 \\
Spirochetes & 1 \\
Unassigned & 1,671 \\
\hline Total & 2,960 \\
\hline
\end{tabular}

Prediction was obtained using Tsetse+Prokaryotes-BLSOM.

Staphylococcus $(n=134)$, Enterococcus $(n=83)$, Wolbachia $(n=56)$, Polaribacter $(n=37)$, and Listeria $(n=31)$. The HGT candidates associated with the genera Wigglesworthia and Sodalis, which are common endosymbionts of the tsetse fly, were not detected using BLSOM. Most tsetse flies are heavily infected with Wigglesworthia; its abundance reaches over $99 \%$ in natural $\mathrm{Gmm}$ populations [10]. The lack of genome sequences associated with Wigglesworthia may support the high quality of tsetse fly genome sequences, since some genome data are contaminated by symbiont genomes, which can lead to the false-positive detection of HGT events [31]. This result also suggests that the symbiosis between Wigglesworthia and the tsetse fly was recent, as suggested by its genome features [32]. Nonetheless, we cannot exclude the possibility of bacterial genome contaminations in the tsetse fly genome since diverse bacteria exist in tsetse fly [10] and their sequences might not have been completely removed during the genome assembly process.

The high frequency of HGT candidates from the genus Bacillus suggests that there was a strong association between the tsetse fly and Bacillus in the past. Members of the genus
Bacillus are ubiquitous in nature and have been isolated from diverse environments such as water, soil, plants, animals, and air [33]. Some species, such as Bacillus thuringiensis, have been well studied as agents of biological control of arthropods [34]. Kaaya and Darji infected several Bacillus species, including $B$. thuringiensis, to the adult $\mathrm{Gmm}$ and found that the mortality of $\mathrm{Gmm}$ was depending on the bacterial species [35], indicating that some Bacillus species may have infected $\mathrm{Gmm}$ persistently without adverse effect on the hosts and served as HGT donors. In fact, in a microbiota analysis of one tsetse fly species, Glossina fuscipes fuscipes, the bacteria belonging to the genus Bacillus were found dominant in a culture-dependent manner [36].

In contrast, there is no report on the relationship between the tsetse fly and Flavobacteria, which was identified as a second dominant donor of HGT candidates in this study (Table 2). Flavobacteria are symbionts in several arthropods [37-43], which indicates a high probability of the proliferation of this group of bacteria in arthropod hosts including tsetse fly. A comparative genome analysis of a flavobacterial symbiont (Blattabacterium strain Bge) in the omnivorous German cockroach (Blattella germanica) suggested that it plays roles in nutrient supply to the host, amino acid catabolism, and nitrogen excretion [41]. Flavobacterial symbionts in the ladybird (Coleomegilla maculata), and coccinellid beetle (Adonia variegata) induce male-killing [38, 39], in which male progeny in infected females die during embryogenesis. This phenomenon is widely recognised in other bacteria, such as Wolbachia, Rickettsia, Arsenophonus, Spiroplasma, and Cardinium [44]. Hurst et al. proposed that two male-killing symbionts cannot coexist at equilibrium in a single host species based on an observational study of the two-spot ladybird (Adalia bipunctata) infected with two symbionts, Rickettsia and Spiroplasma [39]. The presence of Wolbachia in the tsetse fly and HGT elements from Wolbachia in the tsetse fly genome $[12,13]$ may explain the absence of Flavobacteria in current tsetse fly populations.

3.2. HGT Candidates Derived from Wolbachia. We performed a BLASTn analysis to detect HGT candidates derived from Wolbachia. For 38 contigs, we detected sequence homology with Wolbachia sequences based on the criteria described earlier. Of these 38 contigs, we identified 36 as 


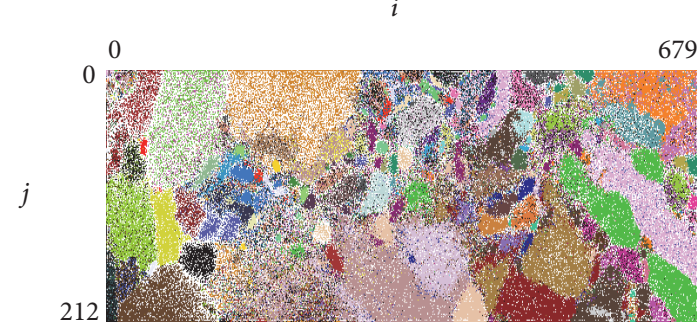

(a)

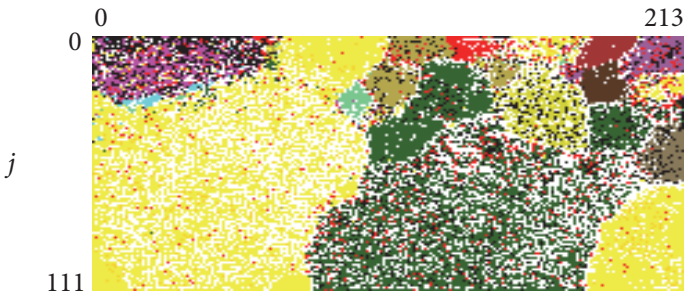

(c)

$i$

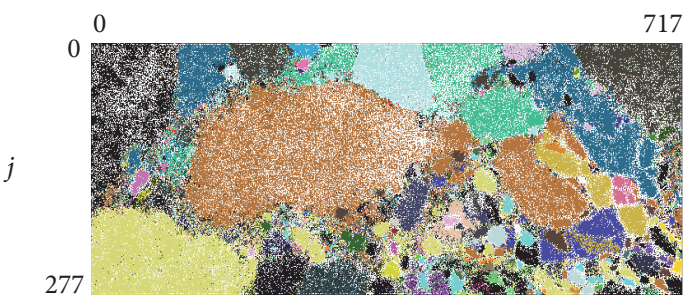

(e)

679

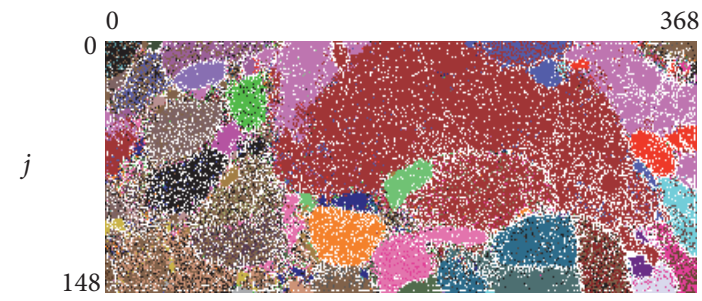

(b)

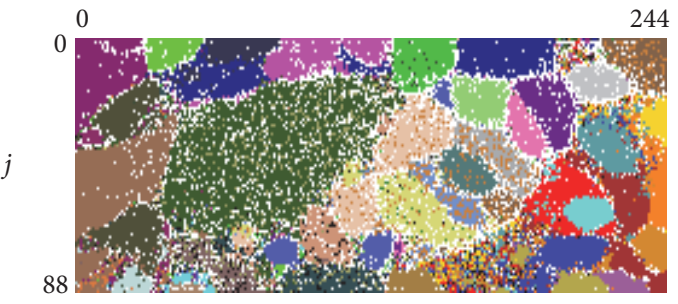

(d)

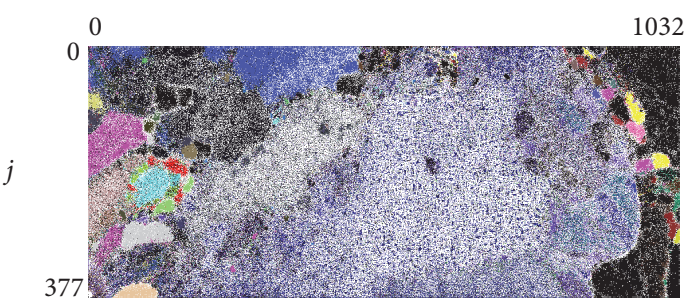

(f)

Figure 3: Genus-BLSOM. (a) Alphaproteobacteria. (b) Bacteroidetes. (c) Epsilonproteobacteria. (d) Euryarchaeota. (e) Firmicutes. (f) Gammaproteobacteria. Lattice points that include sequences from more than one genus are indicated in black, those including no sequences are indicated in white, and those including sequences from a single genus are indicated in individual color.

HGT candidates from Wolbachia using BLSOM, while we assigned the remaining two contigs to the genus Rickettsia using BLSOM. Accordingly, we observed a high level of agreement $(36 / 38=94.7 \%)$ between the two approaches, indicating that the analytical sensitivity of BLSOM is at least comparable to that of BLASTn. These results also suggest that the genetic elements derived from Wolbachia were recently introduced to the tsetse fly genome. Based on a comparison between the BLSOM and BLASTn results, we observed 20 contigs that were only identified as HGT candidates using BLSOM. There may be as-yet unidentified Wolbachia strains, explaining the failure to identify HGT candidates based on sequence homology. In fact, for short regions (i.e., 338, 492, 497, and $497 \mathrm{bp}$ ) of four contigs identified as HGT candidates from Wolbachia only using BLSOM, we detected sequence homology with a Wolbachia endosymbiont of $\mathrm{Gmm}$ (Accession number AWUH01000121) with an $E$-value of $<1 \times$ $10^{-5}$.

Genetic elements related to Wolbachia that were presumably obtained by HGT have been detected in the genomes of multiple arthropod species, including the adzuki bean beetle (Callosobruchus chinensis) [45, 46], a fruit fly (Drosophila ananassae) [47], parasitoid wasps (Nasonia spp.) [47], the pea aphid (Acyrthosiphon pisum) [48], two mosquito species (Aedes aegypti and Aedes mascarensis) [49, 50], and the longicorn beetle (Monochamus alternatus) [51]. Some HGT events could be explained by nuclear-phage recombination, as proposed previously [49], but further studies are needed to determine the specific mechanisms of transfer. Since BLSOM can be used to detect Wolbachia-derived HGTs that exhibit low sequence similarity with known Wolbachia strains, it provides an alternative method with which to explore the mechanisms of HGT. The application of BLSOM to an increasing number of eukaryotic genomes will reveal the diversity and frequency of Wolbachia-derived HGTs in other arthropods, including vectors of medical and veterinary importance.

3.3. Functional Classification of HGT Candidates. We mapped all of the HGT candidates identified using Tsetse+Prokaryotes-BLSOM $(n=11,524)$ to the KEGG pathway. The KO included 317 biological pathways. The predicted pathways were mainly related to "Metabolic pathways" (193 molecules), "Biosynthesis of secondary metabolites" (75 molecules), and "Biosynthesis of antibiotics" (50 molecules). These results suggested that the HGT candidates have the 
TABLE 2: Origins of HGT candidates at the genus level.

\begin{tabular}{|c|c|c|}
\hline Phylum & Genus $^{1}$ & Number of contigs \\
\hline \multirow{7}{*}{ Alphaproteobacteria } & Anaplasma & 4 \\
\hline & Bartonella & 8 \\
\hline & Ehrlichia & 1 \\
\hline & Neorickettsia & 8 \\
\hline & Rickettsia & 5 \\
\hline & Wolbachia & 56 \\
\hline & Unassigned & 8 \\
\hline \multirow{15}{*}{ Bacteroidetes } & Bacteroides & 4 \\
\hline & Cytophaga & 1 \\
\hline & Dyadobacter & 1 \\
\hline & Flavobacteria $^{2}$ & 187 \\
\hline & Flavobacterium & 12 \\
\hline & Kordia & 18 \\
\hline & Leadbetterella & 3 \\
\hline & Mucilaginibacter & 1 \\
\hline & Paludibacter & 9 \\
\hline & Pedobacter & 1 \\
\hline & Polaribacter & 37 \\
\hline & Prevotella & 10 \\
\hline & Psychroflexus & 4 \\
\hline & Spirosoma & 4 \\
\hline & Unassigned & 78 \\
\hline \multirow{20}{*}{ Firmicutes } & Clostridium & 4 \\
\hline & Bacillus & 239 \\
\hline & Enterococcus & 83 \\
\hline & Epulopiscium & 4 \\
\hline & Erysipelothrix & 1 \\
\hline & Geobacillus & 3 \\
\hline & Lactobacillus & 4 \\
\hline & Lactococcus & 1 \\
\hline & Leuconostoc & 17 \\
\hline & Listeria & 31 \\
\hline & Lysinibacillus & 7 \\
\hline & Oenococcus & 1 \\
\hline & Paenibacillus & 1 \\
\hline & Peptoniphilus & 1 \\
\hline & Staphylococcus & 134 \\
\hline & Streptococcus & 9 \\
\hline & Thermoanaerobacter & 3 \\
\hline & Turicibacter & 2 \\
\hline & Veillonella & 3 \\
\hline & Unassigned & 210 \\
\hline \multirow{8}{*}{ Gammaproteobacteria } & Acinetobacter & 4 \\
\hline & Enterobacter & 6 \\
\hline & Escherichia & 1 \\
\hline & Haemophilus & 1 \\
\hline & Shewanella & 1 \\
\hline & Vibrio & 1 \\
\hline & Xylella & 1 \\
\hline & Unassigned & 8 \\
\hline
\end{tabular}

${ }^{1}$ Prediction was obtained using Genus-BLSOM. ${ }^{2}$ Classification to the class level was obtained.

potential to affect a large number of metabolic activities; however, further analyses are essential to demonstrate the active transcription of HGT-acquired genes using transcriptomics or gene-specific reverse transcription-PCR; such analyses can provide initial evidence for the functional importance of HGT-acquired genes [52]. In general, genes transferred to host genomes are pseudogenised via the acquisition of mutations, including insertions and deletions $[46,52,53]$. Unfortunately, since we could not employ RNA sequencing data into our analysis, it is not clear to what extent the detected HGT candidates have been pseudogenised. Nonetheless, active transcription of HGT-acquired genes has been detected in recipient hosts, such as a Wolbachia-derived gene in the Aedes albopictus C6/36 cell line [54]. Moreover, an increasing number of studies suggests that HGT-acquired genes facilitate the establishment of obligate mutualistic relationships between arthropods and their symbionts [55]. Analyses of the functional roles of HGT-acquired genes may improve our understanding of the complex interactions between the tsetse fly, microbes, and pathogens.

\section{Conclusions}

We investigated the use of BLSOM to detect HGT candidates in the tsetse fly genome. Using BLSOM, we successfully detected a number of HGT candidates from diverse bacterial origins. The HGT candidates represented $3.8 \%$ of the tsetse fly genome. The predicted donors of these HGT elements included Wolbachia, a well-known symbiont of the tsetse fly. In addition, using BLSOM, we identified HGT candidates from bacteria that have not previously been associated with the tsetse fly. We observed the HGT candidates from diverse bacteria such as Bacillus and Flavobacteria, suggesting a strong past association between these taxa. In a comparison between BLASTn and BLSOM results for the detection of HGT candidates from Wolbachia, the analytical sensitivity of BLSOM was at least comparable to that of the sequence homology-based approach. Furthermore, BLSOM can be used to detect HGT elements from organisms with low similarity with currently available sequences. These data obtained using BLSOM provide a basis for understanding the coevolutionary history of the tsetse fly and its microbes.

\section{Competing Interests}

The authors declare that there are no competing interests regarding the publication of this paper.

\section{Authors' Contributions}

Ryo Nakao and Takashi Abe contributed equally to this work.

\section{Acknowledgments}

This work was supported by JSPS KAKENHI Grant-in-Aid for Young Scientists (B) (25850195) and (A) (15H05633), for Scientific Research (C) (26330327), and for Scientific Research on Innovative Areas (16H06429, 16K21723, and 16H06431). The computation was done in part with the Earth Simulator of Japan Agency for Marine-Earth Science and Technology. 


\section{References}

[1] G. M. Attardo, C. Lohs, A. Heddi, U. H. Alam, S. Yildirim, and S. Aksoy, "Analysis of milk gland structure and function in Glossina morsitans: milk protein production, symbiont populations and fecundity," Journal of Insect Physiology, vol. 54, no. 8, pp. 1236-1242, 2008.

[2] R. Pais, C. Lohs, Y. Wu, J. Wang, and S. Aksoy, "The obligate mutualist Wigglesworthia glossinidia influences reproduction, digestion, and immunity processes of its host, the tsetse fly," Applied and Environmental Microbiology, vol. 74, no. 19, pp. 5965-5974, 2008.

[3] B. L. Weiss, R. Mouchotte, R. V. M. Rio et al., "Interspecific transfer of bacterial endosymbionts between tsetse fly species: infection establishment and effect on host fitness," Applied and Environmental Microbiology, vol. 72, no. 11, pp. 7013-7021, 2006.

[4] C. Dale and S. C. Welburn, "The endosymbionts of tsetse flies: manipulating host-parasite interactions," International Journal for Parasitology, vol. 31, no. 5-6, pp. 628-631, 2001.

[5] A. Geiger, S. Ravel, R. Frutos, and G. Cuny, "Sodalis glossinidius (Enterobacteriaceae) and vectorial competence of Glossina palpalis gambiensis and Glossina morsitans morsitans for Trypanosoma congolense Savannah type," Current Microbiology, vol. 51, no. 1, pp. 35-40, 2005.

[6] A. Geiger, S. Ravel, T. Mateille et al., "Vector competence of Glossina palpalis gambiensis for Trypanosoma brucei s.l. and genetic diversity of the symbiont Sodalis glossinidius," Molecular Biology and Evolution, vol. 24, no. 1, pp. 102-109, 2007.

[7] J. H. Werren, L. Baldo, and M. E. Clark, "Wolbachia: master manipulators of invertebrate biology," Nature Reviews Microbiology, vol. 6, no. 10, pp. 741-751, 2008.

[8] A. Saridaki and K. Bourtzis, "Wolbachia: more than just a bug in insects genitals," Current Opinion in Microbiology, vol. 13, no. 1, pp. 67-72, 2010.

[9] U. Alam, J. Medlock, C. Brelsfoard et al., "Wolbachia symbiont infections induce strong cytoplasmic incompatibility in the tsetse fly glossina morsitans," PLoS Pathogens, vol. 7, no. 12, Article ID e1002415, 2011.

[10] E. Aksoy, E. L. Telleria, R. Echodu et al., "Analysis of multiple tsetse fly populations in Uganda reveals limited diversity and species-specific gut microbiota," Applied and Environmental Microbiology, vol. 80, no. 14, pp. 4301-4312, 2014.

[11] V. Doudoumis, G. Tsiamis, F. Wamwiri et al., "Detection and characterization of Wolbachia infections in laboratory and natural populations of different species of tsetse flies (genus Glossina)," BMC Microbiology, vol. 12, supplement 1, article S3, 2012.

[12] International Glossina Genome Initiative, "Genome sequence of the tsetse fly (Glossina morsitans): vector of African trypanosomiasis," Science, vol. 344, no. 6182, pp. 380-386, 2014.

[13] C. Brelsfoard, G. Tsiamis, M. Falchetto et al., "Presence of extensive Wolbachia symbiont insertions discovered in the genome of its host Glossina morsitans morsitans," PLoS Neglected Tropical Diseases, vol. 8, no. 4, Article ID e2728, 2014.

[14] O. Adato, N. Ninyo, U. Gophna, and S. Snir, "Detecting horizontal gene transfer between closely related taxa," PLoS Computational Biology, vol. 11, no. 10, Article ID e1004408, 2015.

[15] J. Tamames and A. Moya, "Estimating the extent of horizontal gene transfer in metagenomic sequences," BMC Genomics, vol. 9, article 136, 2008.

[16] S. Kanaya, Y. Yamada, Y. Kudo, and T. Ikemura, "Studies of codon usage and tRNA genes of 18 unicellular organisms and quantification of Bacillus subtilis tRNAs: gene expression level and species-specific diversity of codon usage based on multivariate analysis," Gene, vol. 238, no. 1, pp. 143-155, 1999.

[17] S. Garcia-Vallvé, A. Romeu, and J. Palau, "Horizontal gene transfer in bacterial and archaeal complete genomes," Genome Research, vol. 10, no. 11, pp. 1719-1725, 2000.

[18] Y. Nakamura, T. Itoh, H. Matsuda, and T. Gojobori, "Biased biological functions of horizontally transferred genes in prokaryotic genomes," Nature Genetics, vol. 36, no. 7, pp. 760-766, 2004.

[19] S. Kanaya, M. Kinouchi, T. Abe et al., "Analysis of codon usage diversity of bacterial genes with a self-organizing map (SOM): characterization of horizontally transferred genes with emphasis on the E. coli O157 genome," Gene, vol. 276, no. 1-2, pp. 89-99, 2001.

[20] T. Abe, S. Kanaya, M. Kinouchi, Y. Ichiba, T. Kozuki, and T. Ikemura, "Informatics for unveiling hidden genome signatures," Genome Research, vol. 13, no. 4, pp. 693-702, 2003.

[21] T. Abe, H. Sugawara, M. Kinouchi, S. Kanaya, and T. Ikemura, "Novel phylogenetic studies of genomic sequence fragments derived from uncultured microbe mixtures in environmental and clinical samples," DNA Research, vol. 12, no. 5, pp. 281-290, 2005.

[22] R. Nakao, T. Abe, A. M. Nijhof et al., "A novel approach, based on BLSOMs (Batch Learning Self-Organizing Maps), to the microbiome analysis of ticks," The ISME Journal, vol. 7, no. 5, pp. 1003-1015, 2013.

[23] S. Karlin, "Global dinucleotide signatures and analysis of genomic heterogeneity," Current Opinion in Microbiology, vol. 1, no. 5, pp. 598-610, 1998.

[24] R. Grantham, C. Gautier, M. Gouy, R. Mercier, and A. Pavé, "Codon catalog usage and the genome hypothesis," Nucleic Acids Research, vol. 8, no. 1, pp. r49-r62, 1980.

[25] T. Kohonen, "Self-organized formation of topologically correct feature maps," Biological Cybernetics, vol. 43, no. 1, pp. 59-69, 1982.

[26] T. Kohonen, E. Oja, O. Simula, A. Visa, and J. Kangas, "Engineering applications of the self-organizing map," Proceedings of the IEEE, vol. 84, no. 10, pp. 1358-1383, 1996.

[27] S. Kanaya, Y. Kudo, T. Abe, T. Okazaki, C. D. Carpio, and T. Ikemura, "Gene classification by self-organization mapping of codon usage in bacteria with completely sequenced genome," Genome Informatics, vol. 9, pp. 369-371, 1998.

[28] M. Kanehisa and S. Goto, "KEGG: kyoto encyclopedia of genes and genomes," Nucleic Acids Research, vol. 28, no. 1, pp. 27-30, 2000.

[29] Y. Moriya, M. Itoh, S. Okuda, A. C. Yoshizawa, and M. Kanehisa, "KAAS: an automatic genome annotation and pathway reconstruction server," Nucleic Acids Research, vol. 35, no. 2, pp. W182-W185, 2007.

[30] J. R. Brown, "Ancient horizontal gene transfer," Nature Reviews Genetics, vol. 4, no. 2, pp. 121-132, 2003.

[31] I. I. Artamonova and A. R. Mushegian, "Genome sequence analysis indicates that the model eukaryote Nematostella vectensis harbors bacterial consorts," Applied and Environmental Microbiology, vol. 79, no. 22, pp. 6868-6873, 2013.

[32] L. Akman, A. Yamashita, H. Watanabe et al., "Genome sequence of the endocellular obligate symbiont of tsetse flies, Wigglesworthia glossinidia," Nature Genetics, vol. 32, no. 3, pp. 402-407, 2002.

[33] M. Pignatelli, A. Moya, and J. Tamames, "EnvDB, a database for describing the environmental distribution of prokaryotic taxa," 
Environmental Microbiology Reports, vol. 1, no. 3, pp. 191-197, 2009.

[34] A. Bravo, S. Likitvivatanavong, S. S. Gill, and M. Soberón, "Bacillus thuringiensis: a story of a successful bioinsecticide," Insect Biochemistry and Molecular Biology, vol. 41, no. 7, pp. 423431, 2011.

[35] G. P. Kaaya and N. Darji, "Mortality in adult tsetse, Glossina morsitans morsitans, caused by entomopathogenic bacteria," Journal of Invertebrate Pathology, vol. 54, no. 1, pp. 32-38, 1989.

[36] J. M. Lindh and M. J. Lehane, "The tsetse fly Glossina fuscipes fuscipes (Diptera: Glossina) harbours a surprising diversity of bacteria other than symbionts," Antonie van Leeuwenhoek, International Journal of General and Molecular Microbiology, vol. 99, no. 3, pp. 711-720, 2011.

[37] C. Bandi, G. Damiani, L. Magrassi, A. Grigolo, R. Fani, and L. Sacchi, "Flavobacteria as intracellular symbionts in cockroaches," Proceedings of the Royal Society B: Biological Sciences, vol. 257, no. 1348, pp. 43-48, 1994.

[38] G. D. D. Hurst, T. C. Hammarton, C. Bandi, T. M. O. Majerus, D. Bertrand, and M. E. N. Majerus, "The diversity of inherited parasites of insects: the male-killing agent of the ladybird beetle Coleomegilla maculata is a member of the Flavobacteria," Genetical Research, vol. 70, no. 1, pp. 1-6, 1997.

[39] G. D. D. Hurst, C. Bandi, L. Sacchi et al., "Adonia variegata (Coleoptera: Coccinellidae) bears maternally inherited Flavobacteria that kill males only," Parasitology, vol. 118, no. 2, pp. 125-134, 1999.

[40] S. Van Borm, A. Buschinger, J. J. Boomsma, and J. Billen, "Tetraponera ants have gut symbionts related to nitrogenfixing root-nodule bacteria," Proceedings of the Royal Society B: Biological Sciences, vol. 269, no. 1504, pp. 2023-2027, 2002.

[41] M. J. López-Sánchez, A. Neef, J. Peretó et al., "Evolutionary convergence and nitrogen metabolism in Blattabacterium strain Bge, primary endosymbiont of the cockroach Blattella germanica," PLoS Genetics, vol. 5, no. 11, Article ID e1000721, 2009.

[42] Y. Matsuura, R. Koga, N. Nikoh, X.-Y. Meng, S. Hanada, and T. Fukatsu, "Huge symbiotic organs in giant scale insects of the genus Drosicha (Coccoidea: Monophlebidae) harbor flavobacterial and enterobacterial endosymbionts," Zoological Science, vol. 26, no. 7, pp. 448-456, 2009.

[43] M. Rosenblueth, L. Sayavedra, H. Sámano-Sánchez, A. Roth, and E. Martínez-Romero, "Evolutionary relationships of flavobacterial and enterobacterial endosymbionts with their scale insect hosts (Hemiptera: Coccoidea)," Journal of Evolutionary Biology, vol. 25, no. 11, pp. 2357-2368, 2012.

[44] D. Kageyama, S. Narita, and M. Watanabe, "Insect sex determination manipulated by their endosymbionts: incidences, mechanisms and implications," Insects, vol. 3, no. 1, pp. 161-199, 2012.

[45] N. Kondo, N. Nikoh, N. Ijichi, M. Shimada, and T. Fukatsu, "Genome fragment of Wolbachia endosymbiont transferred to $\mathrm{X}$ chromosome of host insect," Proceedings of the National Academy of Sciences of the United States of America, vol. 99, no. 22, pp. 14280-14285, 2002.

[46] N. Nikoh, K. Tanaka, F. Shibata et al., "Wolbachia genome integrated in an insect chromosome: evolution and fate of laterally transferred endosymbiont genes," Genome Research, vol. 18, no. 2, pp. 272-280, 2008.

[47] J. C. Dunning Hotopp, M. E. Clark, D. C. S. G. Oliveira et al., "Widespread lateral gene transfer from intracellular bacteria to multicellular eukaryotes," Science, vol. 317, no. 5845, pp. 17531756, 2007.
[48] N. Nikoh and A. Nakabachi, "Aphids acquired symbiotic genes via lateral gene transfer," BMC Biology, vol. 7, article no. 12, 2009.

[49] L. Klasson, Z. Kambris, P. E. Cook, T. Walker, and S. P. Sinkins, "Horizontal gene transfer between Wolbachia and the mosquito Aedes aegypti," BMC Genomics, vol. 10, article no. 33, 2009.

[50] M. Woolfit, I. Iturbe-Ormaetxe, E. A. McGraw, and S. L. O’Neill, "An ancient horizontal gene transfer between mosquito and the endosymbiotic bacterium Wolbachia pipientis," Molecular Biology and Evolution, vol. 26, no. 2, pp. 367-374, 2009.

[51] T. Aikawa, H. Anbutsu, N. Nikoh, T. Kikuchi, F. Shibata, and T. Fukatsu, "Longicorn beetle that vectors pinewood nematode carries many Wolbachia genes on an autosome," Proceedings of the Royal Society B: Biological Sciences, vol. 276, no. 1674, pp. 3791-3798, 2009.

[52] S. M. Degnan, "Think laterally: horizontal gene transfer from symbiotic microbes may extend the phenotype of marine sessile hosts," Frontiers in Microbiology, vol. 5, article 638, 2014.

[53] V. Doudoumis, U. Alam, E. Aksoy et al., "Tsetse-Wolbachia symbiosis: comes of age and has great potential for pest and disease control," Journal of Invertebrate Pathology, vol. 112, no. 1, pp. S94-S103, 2013.

[54] Q. Hou, J. He, J. Yu et al., "A case of horizontal gene transfer from Wolbachia to Aedes albopictus C6/36 cell line," Mobile Genetic Elements, vol. 4, no. 2, Article ID e28914, 2014.

[55] A. Nakabachi, "Horizontal gene transfers in insects," Current Opinion in Insect Science, vol. 7, article no. 113, pp. 24-29, 2015. 

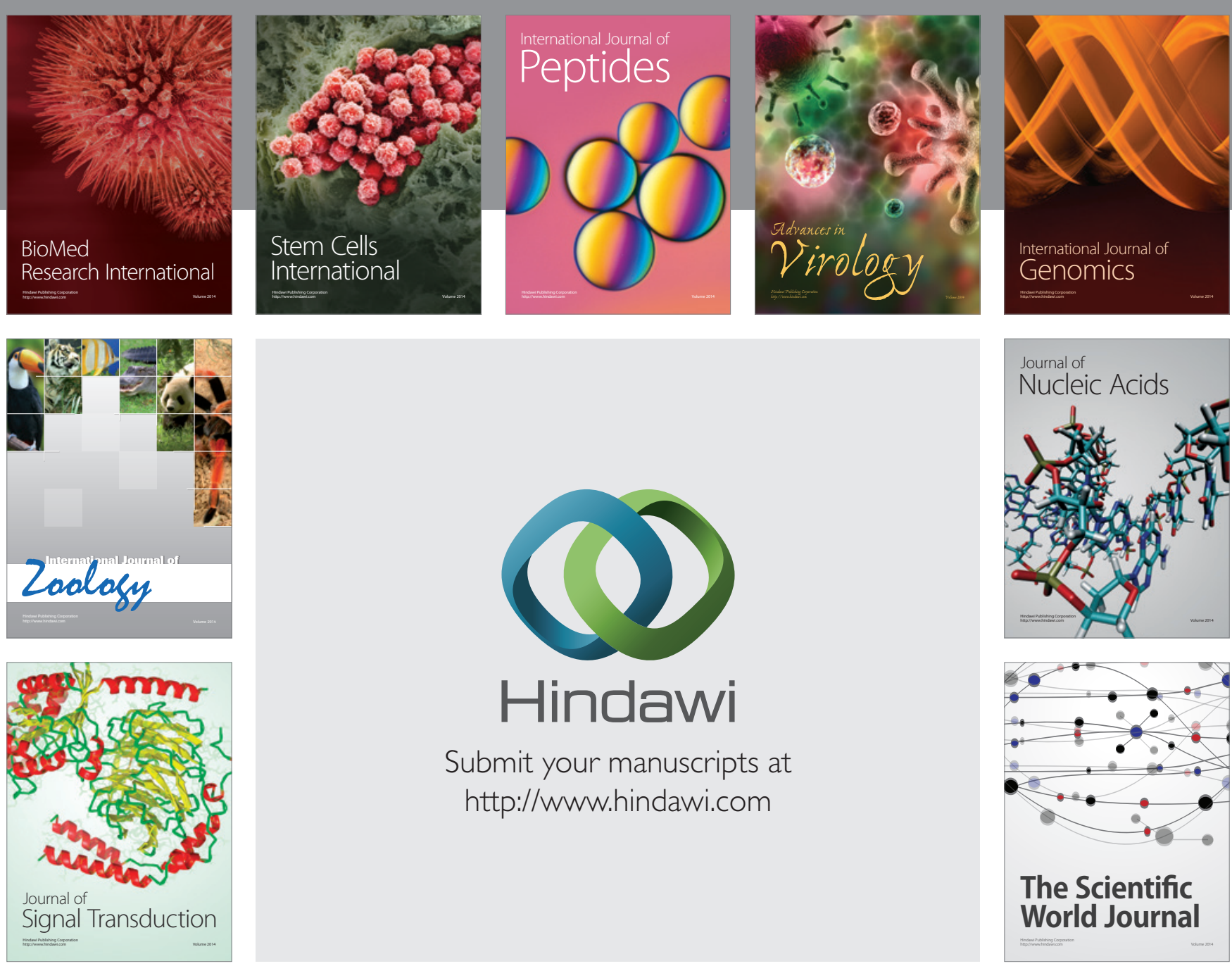

Submit your manuscripts at

http://www.hindawi.com
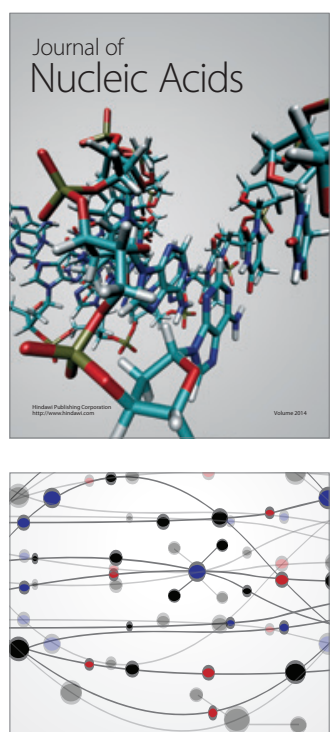

The Scientific World Journal
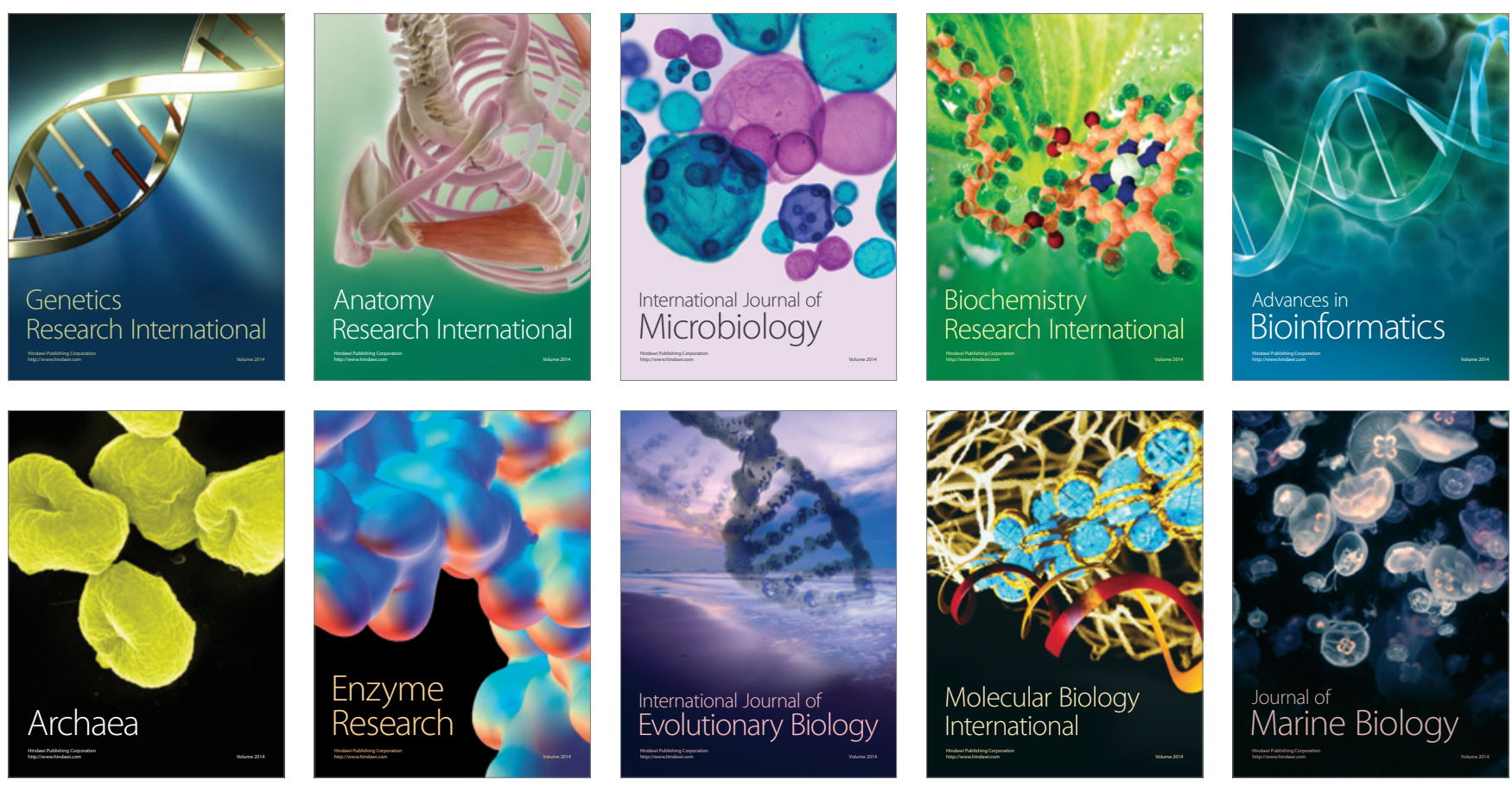